\title{
Environmental Real Time Imaging with 200kV FE Aberration-corrected Analytical Scanning Transmission Electron Microscope (ESTEM) System with an Open Window Gas Injection
}

\author{
H. Inada, ${ }^{1}$ H. Kikuchi, ${ }^{1}$ A. Hanawa, ${ }^{1}$ Y. Suzuki, ${ }^{1}$ M. Shirai, ${ }^{1}$ and K. Nakamura ${ }^{1}$
}

1. Science \& Medical Systems Business Group, Hitachi High-Technologies Corp., Hitachinaka, Ibaraki, Japan

Catalyst or fuel cells, observation of the behavior is necessary to understand the degradation process in real environment (in-situ) with transmission electron microscope (TEM). On the other hand, we have reported evaluation of environmental real time scanning transmission electron microscope (STEM) imaging with a probe-forming aberration corrector equipped analytical $200 \mathrm{kV}$ cold field emission (CFE) TEM (Hitachi HF5000, Figure 1(a)) [1][2][3]. The base microscope is capable of TEM, STEM imaging with bright field (BF), annular dark field (DF) detectors, and secondary electron (SE) imaging. The probe-forming aberration corrector with automated correction of up to third order aberrations allows users to obtain aberration-free STEM illumination optics with minimized effort.

For in-situ operation, we have modified a standard vacuum system suitable for high gas pressure configuration with differential pumping apertures (orifices) and additional turbo-molecular pump (TMP) to improve the evacuation capability in order to keep the gun pressure low enough to operate CFE (Figure 1(b)) [1][4]. This microscope enables to record three scanning image signal simultaneously SE, BF and DF-STEM in TV scan rate. This FE-TEM has a capable of switching standard TEM/STEM analytical condition and insitu operation mode.

Figure 2 (a) shows a GUI of in-situ operation mode controller, which provides user friendliness operation of environmental STEM and TEM vacuum operation as well as gas injection both needle gas pipe and gas injection filament heating holder. Because this TEM has a wider dynamic range scanning capability, it allows to operate a magnification of $\times 20$ which is corresponding to $1,100 \mu \mathrm{m} \times 1,100 \mu \mathrm{m}$ field of view SE image, shows a part of filament heater and gas injection nozzle on the holder. Figure 3 shows, on the other hand, atomic resolution simultaneously acquired DF-STEM and SE images of $\mathrm{Au} / \mathrm{CeO}_{2}$ (gold particle sitting on a $\mathrm{CeO}_{2}$ support), a size of FOV is $18 \mathrm{~nm} \times 18 \mathrm{~nm}$. These images are taken with open window MEMS heating holder so that E-T detector above the specimen is able to detect SE signal. SE, which provides wellknown surface topographic information may derive degradation and/or activation of catalysts [5]. This wide magnification range moreover opens possibility of variety of information. In the presentation we will show newly developed back-scattering electron filter for E-T detector equipped with this Cs-corrected TEM/STEM.

\section{References:}

[1] H Inada et al, Microsc. Microanal. 23 (2017), p. 918.

[2] H Inada et al, Microsc. Microanal. 22 (2016), p. 32.

[3] H Inada et al, European Microsc. Congress. 1 (2016), p. 334.

[4] H Matsumoto et al, Microsc. Microanal. 27(7) (2013), p. 11.

[5] Y Zhu et al, Nature Mater 8 (2009), p. 808. 
(a)

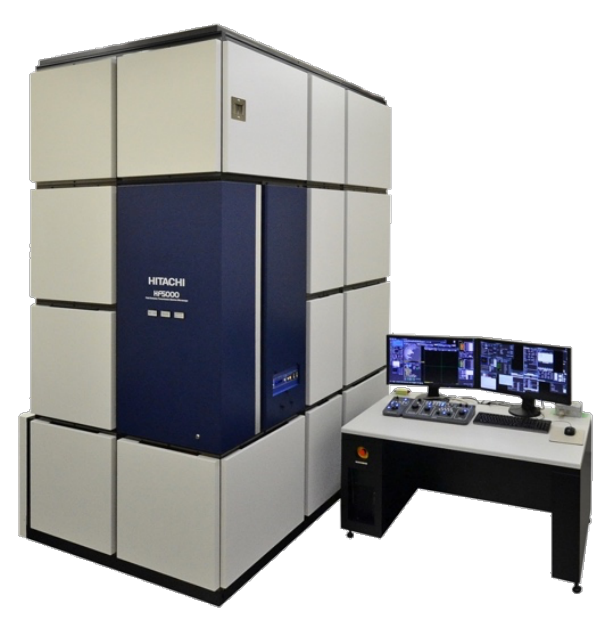

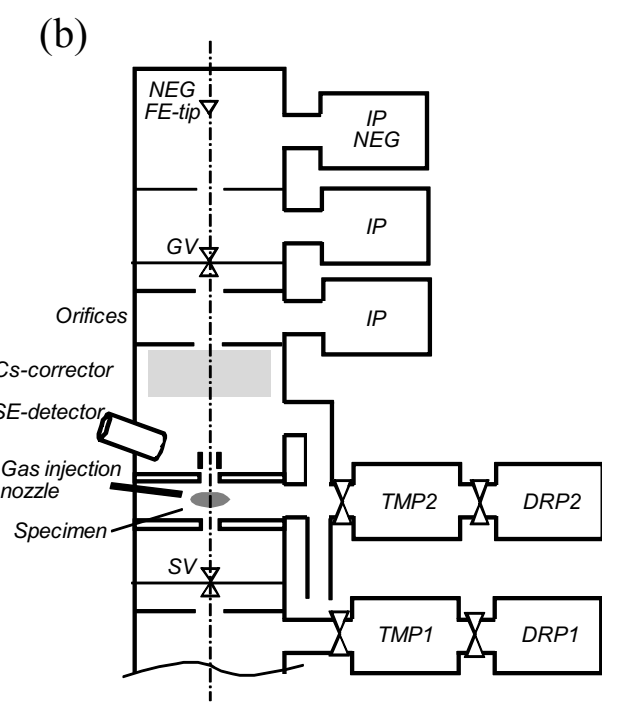

Figure 1. (a) Overview of Hitachi HF5000 200kV Cs-corrected CFE TEM/STEM And (b) a Schematic diagram of differential pumping system suitable for environmental TEM \& STEM
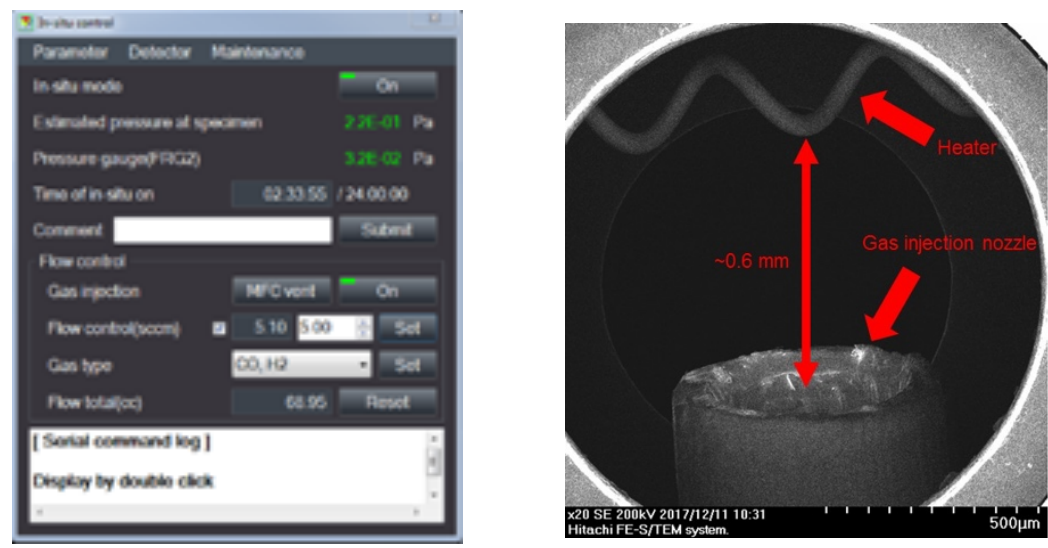

Figure 2. Left: GUI image of "in-situ mode" window to control gas injection and Right: large FOV STEM image of gas injection heating holder (magnification of 20) in the gap of the FE-TEM pole piece.
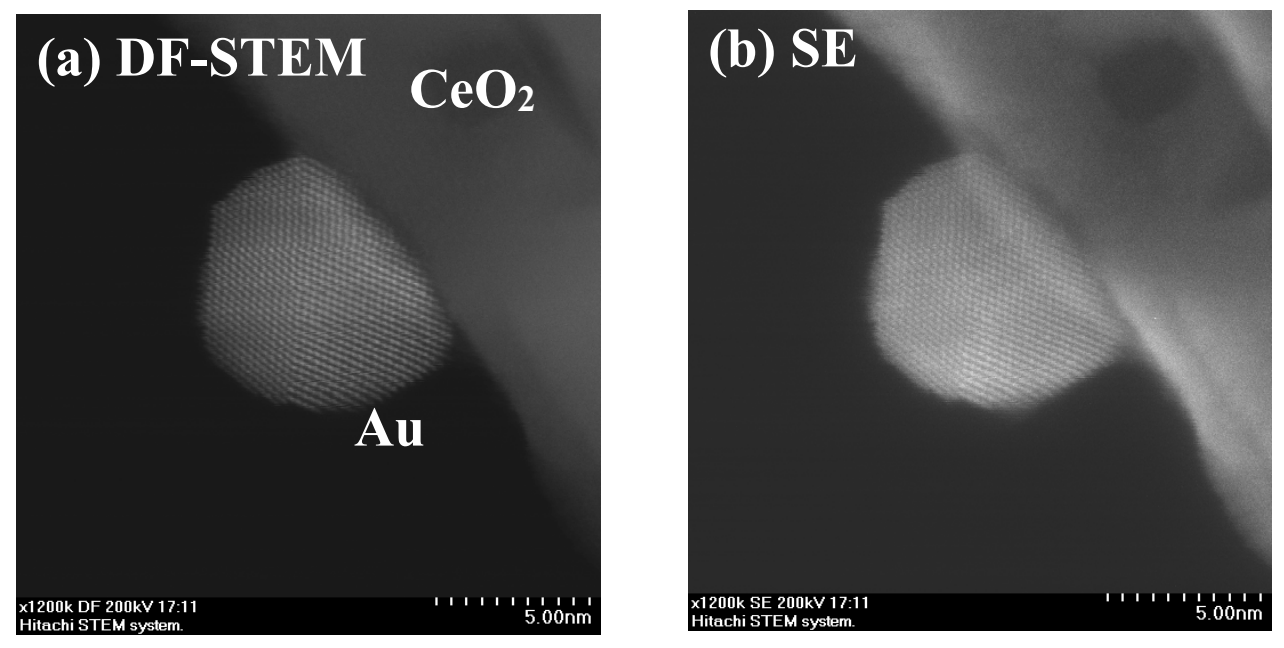

Figure 3. High-resolution simultaneously acquiring DF-STEM (a) and SE (b) images of $\mathrm{Au} / \mathrm{CeO}_{2}$ catalyst on MEMS heating holder. 